\title{
FISHER INFORMATION MATRIX FOR CROVELLI'S AND GAMMA BETA II BIVARIATE DISTRIBUTIONS
}

\author{
Ana Paula Coelho Madeira SILVA ${ }^{1}$ \\ Adélia Conceição DINIZ ${ }^{1}$
}

- ABSTRACT: In this paper, a system of nonlinear equations for the maximum likelihood estimators as wel as the exact forms of the Fisher information matrix for Crovelli's bivariate gamma distribution and bivariate gamma beta distribution of the second kind are determined. An application of the results to the rainfall data from the city of Passo Fundo are provided.

KEYWORDS: Bivariate distributions; Hessian matrix; Hurwitz zeta function; maximum likelihood estimators.

\section{Introduction}

Motivaded by their crescent use, especially in the analysis of non normal data, several bivariate gamma models can be found in literature, see, for example, Balakrishnan and Lai (2009). Among all bivariate gamma models in literature, we have the Crovelli's and bivariate gamma beta of the second kind distributions, whose joint density probability functions (pdf) are given, respectively, by

$$
f(x, y)=\left\{\begin{array}{lll}
\alpha \beta e^{-\alpha x}\left[1-e^{-\beta y}\right] & \text { if } & 0 \leq \frac{\beta}{\alpha} y \leq x \quad(I) \\
\alpha \beta e^{-\beta y}\left[1-e^{-\alpha x}\right] & \text { if } & 0 \leq \frac{\alpha}{\beta} x \leq y \quad(I I)
\end{array}\right.
$$

for $x, y, \alpha, \beta>0$, and

$$
f(x, y ; a, c, \alpha, \beta)=K x^{\alpha-1} y^{\beta-1} e^{-(a x+c x y)}
$$

\footnotetext{
${ }^{1}$ Universidade Federal de São João del Rei - UFSJ, Campus Sete Lagoas, Departamento de Ciências Exatas e Biológicas, CEP: 35702-031, Sete Lagoas, MG, Brasil. E-mail: anapaula@ufsj.edu.br; adelia@ufsj.edu.br 
for $x, y, \alpha, \beta, a, c>0$, where $K$ is the normalization constant defined by

$$
K=\frac{c^{\beta} a^{\alpha-\beta}}{\Gamma(\beta) \Gamma(\alpha-\beta)}
$$

It can be seen in Silva et al., (2013a, 2013b) that the exact distributions of the variables $U=X+Y, P=X Y$ and $Q=X /(X+Y)$ when $X$ and $Y$ follow (1) and (2) were deducted and successfully implemented on modeling hydrological processes.

The aim of this work is to calculate the Fisher information matrices (FIM) corresponding to models (1) and (2), respectively. For a given observation $(x, y)$ the FIM is defined by

$$
\left(I_{j k}\right)=\left\{E\left(-\frac{\partial^{2} \ln L(\boldsymbol{\theta})}{\partial \theta_{j} \partial \theta_{k}}\right)\right\}
$$

for $j=1,2, \ldots, p$ and $k=1,2, \ldots, p$, where $L(\boldsymbol{\theta})=f(x, y)$ and $\boldsymbol{\theta}=\left(\theta_{1}, \theta_{2}, \ldots \theta_{p}\right)$ are the parameters of the pdf $f$.

The information matrix plays a significant role in the statistical inference in connection with estimation, sufficiency and properties of variances of estimators (COX and HINKLEY, 1974). It is related to a way of measuring the amount of information that an observable random variable $X$ carries about an unknown parameter $\theta_{j}$ of a distribution that models $X$. Fisher's information provides a limitation for the variance of the non-additive variance estimator uniformly from $\theta_{j}$. The FIM is used to calculate the covariance matrices associated with maximumlikelihood estimators. It can also be used in the formulation of test statistics, such as the Wald test (LIU et al., 2014); for intervals estimation and hypothesis tests on the distribution parameters (BARROS et al., 2017; LOUZADA et al. , 2018), and it is also applicable to unknown systematic errors (FISCHER, 2018).

Deriving the explicit formulae for the elements of the Fisher information matrix for the complicated distributions have attracted the interest of several authors (see NADARAJAH, 2006a; PARK and KIM, 2007; ALI and NADARAJAH, 2007; GUPTA and NADARAJAH, 2007; QIAN, 2012). In a tutorial presented by Ly et al. (2017) the authors illustrate the use of Fisher's information in three different paradigms: in the frequentist, Bayesian, and paradigm of information theory. A general theory regarding information quantities such as the Fisher score, the Renyi entropy, and the Fisher information of a random vector in $R^{n}$ is presented in $\mathrm{Lv}$ (2017).

A system of nonlinear equations for the maximum likelihood estimators and exacts forms of the Fisher information matrix are derived in Section 2 and Section 3, for Crovelli's bivariate gamma distribution and bivariate gamma beta of the second kind distribution, respectively. An application of the results to rainfall data from the city of Passo Fundo is illustrated in Section 4.

The calculations involve some special functions such as the Gamma function defined by

Rev. Bras. Biom., Lavras, v.39, n.2, p.350-361, 2021 - doi: 10.28951/rbb.v39i2.476 


$$
\Gamma(s)=\int_{0}^{\infty} t^{s-1} e^{-t} d t
$$

the psi function defined by

$$
\psi(s)=\frac{d}{d s}[\ln \Gamma(s)]
$$

the Riemann zeta function defined by

$$
\zeta(s)=\sum_{n=1}^{\infty} \frac{1}{n^{s}}
$$

the Hurwitz zeta function defined by

$$
\zeta(s, a)=\sum_{n=0}^{\infty} \frac{1}{(n+a)^{s}}, a>0
$$

and the identity

$$
\frac{d}{d s} \psi(s)=\sum_{n=0}^{\infty} \frac{1}{(n+s)^{2}}
$$

The properties of these special functions can be found in Oldham et al., (2009) and Beals and Wong (2010).

\section{Crovelli's bivariate gamma distribution}

In this section we present a system of nonlinear equations for the maximum likelihood estimators and an exact form of the Fisher information matrix for Crovelli's bivariate gamma distribution. In some papers, such as (GUPTA and NADARAJAH, 2007; NADARAJAH, 2006a, 2006b; ALI and NADARAJAH, 2007), the authors have determined the FIM for probability density functions(pdf's) considering only one sample point in the pdf's sample spaces. In this paper we've calculated the FIM for Crovelli's and gamma beta II bivariate distributions for a set of $n$ observations, $\left(x_{1}, y_{1}\right), \ldots,\left(x_{n}, y_{n}\right)$, in their respectively sample spaces.

\subsection{Maximum likelihood estimators}

Let $\left(x_{1}, y_{1}\right), \ldots,\left(x_{n}, y_{n}\right)$ be observations having the joint probability density function given by (1). Let's reorder it such that $\left(x_{1}, y_{1}\right), \cdots,\left(x_{k}, y_{k}\right)$ are in the region $(I)$ and the last ones $\left(x_{k+1}, y_{k+1}\right) \cdots,\left(x_{n}, y_{n}\right)$ are in the region $(I I)$ of $(1)$, as shown in Figure 1.

Then, the likelihood function, $L(\alpha, \beta)=\prod_{i=1}^{n} f\left(x_{i}, y_{i}\right)$, is given by 


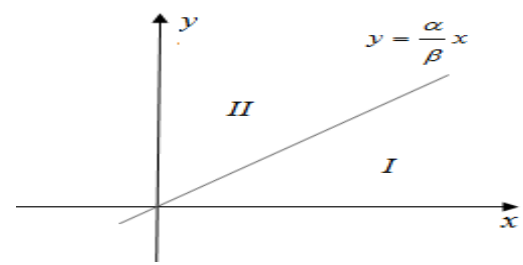

Figure 1 - Region of integration.

$$
L(\alpha, \beta)= \begin{cases}\prod_{i=1}^{k} \alpha \beta e^{-\alpha x_{i}}\left[1-e^{-\beta y_{i}}\right] & , \quad 0<\frac{\beta}{\alpha} y_{i}<x_{i} \\ \prod_{j=k+1}^{n} \alpha \beta e^{-\beta y_{j}}\left[1-e^{-\alpha x_{j}}\right] & , \quad 0<\frac{\alpha}{\beta} x_{j}<y_{j}\end{cases}
$$

Consequently, the log-likelihood function is given by

$\ln L=\left\{\begin{array}{cc}k \ln \alpha+k \ln \beta-\alpha \sum_{i=1}^{k} x_{i}+\sum_{i=1}^{k} \ln \left[1-e^{-\beta y_{i}}\right] & 0<\frac{\beta}{\alpha} y_{i}<x_{i} \\ (n-k) \ln \alpha+(n-k) \ln \beta-\beta \sum_{j=k+1}^{n} y_{j}+\sum_{j=k+1}^{n} \ln \left[1-e^{-\alpha x_{j}}\right] & , 0<\frac{\alpha}{\beta} x_{j}<y_{j}\end{array}\right.$

The first-order derivatives of the log-likelihood function are

$$
\begin{gathered}
\frac{\partial \ln L}{\partial \alpha}=\left\{\begin{array}{cl}
\frac{k}{\alpha}-\sum_{i=1}^{k} x_{i} & , \quad 0<\frac{\beta}{\alpha} y_{i}<x_{i} \\
\frac{n-k}{\alpha}+\sum_{j=k+1}^{n} \frac{x_{j} e^{-\alpha x_{j}}}{1-e^{-\alpha x_{j}}} & , \quad 0<\frac{\alpha}{\beta} x_{j}<y_{j}
\end{array}\right. \\
\frac{\partial \ln L}{\partial \beta}=\left\{\begin{array}{cl}
\frac{k}{\beta}+\sum_{i=1}^{k} \frac{y_{i} e^{-\beta y_{i}}}{1-e^{-\beta y_{i}}} \quad, \quad 0<\frac{\beta}{\alpha} y_{i}<x_{i} \\
\frac{n-k}{\beta}-\sum_{j=k+1}^{n} y_{j} & , 0<\frac{\alpha}{\beta} x_{j}<y_{j}
\end{array}\right.
\end{gathered}
$$

Hence, the maximum likelihood estimators (MLE's) of parameters $(\alpha, \beta)$ are obtained by solving

$$
\frac{\partial \ln L}{\partial \alpha}=0 \quad \text { and } \quad \frac{\partial \ln L}{\partial \beta}=0
$$

which results in the following system of nonlinear equations 


$$
\left\{\begin{array}{l}
\frac{n}{\alpha}-\sum_{i=1}^{n} x_{i}+\sum_{j=k+1}^{n} \frac{x_{j}}{1-e^{-\alpha x_{j}}}=0 \\
\frac{n}{\beta}-\sum_{i=1}^{n} y_{i}+\sum_{i=1}^{k} \frac{y_{i}}{1-e^{-\beta y_{i}}}=0
\end{array} .\right.
$$

\subsection{Fisher information matrix}

The Fisher information matrix for model (1) is obtained by taking expectation on the Hessian matrix of the log-likelihood function, as following:

$$
I=\left(\begin{array}{cc}
E\left(-\frac{\partial^{2} \ln L}{\partial \alpha^{2}}\right) & E\left(-\frac{\partial^{2} \ln L}{\partial \alpha \partial \beta}\right) \\
E\left(-\frac{\partial^{2} \ln L}{\partial \beta \partial \alpha}\right) & E\left(-\frac{\partial^{2} \ln L}{\partial \beta^{2}}\right)
\end{array}\right)
$$

The second-order derivatives are given by

$$
\begin{aligned}
& \frac{\partial^{2} \ln L}{\partial \alpha^{2}}=\left\{\begin{array}{cc}
-\frac{k}{\alpha^{2}} & 0<\frac{\beta}{\alpha} y_{i}<x_{i} \\
-\frac{n-k}{\alpha^{2}}-\sum_{j=k+1}^{n} \frac{x_{j}^{2} e^{-\alpha x_{j}}}{\left(1-e^{-\alpha x_{j}}\right)^{2}} & , \quad 0<\frac{\alpha}{\beta} x_{j}<y_{j}
\end{array}\right. \\
& \frac{\partial^{2} \ln L}{\partial \alpha \partial \beta}=\frac{\partial^{2} \ln L}{\partial \beta \partial \alpha}=0 . \\
& \frac{\partial^{2} \ln L}{\partial \beta^{2}}=\left\{\begin{array}{cc}
-\frac{k}{\beta^{2}}-\sum_{i=1}^{k} \frac{y_{i}^{2} e^{-\beta y_{i}}}{\left(1-e^{-\beta y_{i}}\right)^{2}} \quad, \quad 0<\frac{\beta}{\alpha} y_{i}<x_{i} \\
-\frac{n}{\beta^{2}} \quad & 0<\frac{\alpha}{\beta} x_{j}<y_{j}
\end{array}\right.
\end{aligned}
$$

The expectations are given by

$$
\begin{aligned}
E\left(-\frac{\partial^{2} \ln L}{\partial \alpha^{2}}\right) & =\frac{1}{2}\left(\frac{k}{\alpha^{2}}\right)+\frac{1}{2}\left(\frac{n-k}{\alpha^{2}}\right)+\alpha \beta \sum_{j=k+1}^{n} \int_{0}^{\infty} \int_{\frac{\alpha x_{j}}{\beta}}^{\infty} \frac{x_{j}^{2} e^{-\alpha x_{j}} e^{-\beta y_{j}}}{1-e^{-\alpha x_{j}}} d y_{j} d x_{j} \\
& =\frac{1}{2} \frac{n}{\alpha^{2}}+\alpha \beta \sum_{j=k+1}^{n} \int_{0}^{\infty}-\left.\frac{1}{\beta} \frac{x_{j}^{2} e^{-\alpha x_{j}}}{1-e^{-\alpha x_{j}}} e^{-\beta y_{j}}\right|_{\frac{\alpha x_{j}}{\beta}} ^{\infty} d x_{j} \\
& =\frac{1}{2} \frac{n}{\alpha^{2}}+\alpha \sum_{j=k+1}^{n} \int_{0}^{\infty} \frac{x_{j}^{2} e^{-2 \alpha x_{j}}}{1-e^{-\alpha x_{j}}} d x_{j}
\end{aligned}
$$


Each one of the integrals

$$
I_{j}=\int_{0}^{\infty} \frac{x_{j}^{2} e^{-2 \alpha x_{j}}}{1-e^{-\alpha x_{j}}} d x_{j}, j=k+1, \ldots, n,
$$

can be calculated by considering the geometric series representation of the function $\frac{x_{j}^{2} e^{-2 \alpha x_{j}}}{1-e^{-\alpha x_{j}}}$. In fact, putting $a=x_{j}^{2} e^{-2 \alpha x_{j}}$ and $r=e^{-\alpha x_{j}}=\frac{1}{e^{\alpha x_{j}}}$, we conclude that the ratio $r$ satisfies the condition $|r|<1$, for all $x_{j}>0$. Then, the geometric series converges to

$$
\frac{a}{1-r}=\sum_{n=1}^{\infty} a r^{n-1}
$$

so that we have

$$
\begin{aligned}
\frac{x_{j}^{2} e^{-2 \alpha x_{j}}}{1-e^{-\alpha x_{j}}} & =\sum_{n=1}^{\infty} x_{j}^{2} e^{-2 \alpha x_{j}}\left(e^{-\alpha x_{j}}\right)^{n-1} \\
& =\sum_{n=1}^{\infty} x_{j}^{2} e^{-(n+1) \alpha x_{j}}
\end{aligned}
$$

Now, each $I_{j}$ can be calculated as following

$$
\begin{aligned}
I_{j} & =\sum_{n=1}^{\infty} \int_{0}^{\infty} x_{j}^{2} e^{-(n+1) \alpha x_{j}} d x_{j} \\
& =\sum_{n=1}^{\infty} \frac{1}{(n+1)^{3} \alpha^{3}} \int_{0}^{\infty} t_{j}^{2} e^{-t_{j}} d t_{j} \\
& =\frac{2}{\alpha^{3}}[\zeta(3)-1]
\end{aligned}
$$

Hence, the equation (10) can be rewritten as

$$
\begin{aligned}
E\left(-\frac{\partial^{2} \ln L}{\partial \alpha^{2}}\right) & =\frac{1}{2} \frac{n}{\alpha^{2}}+\alpha \sum_{j=k+1}^{n} \frac{2}{\alpha^{3}}[\zeta(3)-1] \\
& =\frac{1}{2} \frac{n}{\alpha^{2}}+\frac{2(n-k)}{\alpha^{2}}[\zeta(3)-1] \\
& =\frac{1}{\alpha^{2}}\left\{\frac{n}{2}+2(n-k)[\zeta(3)-1]\right\}
\end{aligned}
$$

Using similar calculations, we can show that

$$
E\left(-\frac{\partial^{2} \ln L}{\partial \beta^{2}}\right)=\frac{1}{\beta^{2}}\left\{\frac{n}{2}+2(n-k)[\zeta(3)-1]\right\}
$$


Consequently, we can express the FIM as

$$
I=\left(\begin{array}{cc}
\frac{1}{\alpha^{2}}\left\{\frac{n}{2}+2(n-k)[\zeta(3)-1]\right\} & 0 \\
0 & \frac{1}{\beta^{2}}\left\{\frac{n}{2}+2(n-k)[\zeta(3)-1]\right\}
\end{array}\right)
$$

\section{Gamma beta II bivariate distribution}

In this section we present a system of nonlinear equations for the maximum likelihood estimators and an exact form of the Fisher information matrix for the gamma beta II bivariate distribution.

\subsection{Maximum likelihood estimators}

Let $\left(x_{1}, y_{1}\right),\left(x_{2}, y_{2}\right), \ldots,\left(x_{n}, y_{n}\right)$ be a random sample of the random variable $(X, Y)$ with joint distribution expressed by (2) from parameters $a, c, \alpha$ and $\beta$. The likelihood function is given by

$$
L(a, c, \alpha, \beta)=\prod_{i=1}^{n} f\left(x_{i}, y_{i}\right)=\prod_{i=1}^{n} K x_{i}^{\alpha-1} y_{i}^{\beta-1} e^{\left[-\left(a x_{i}+c x_{i} y_{i}\right)\right]}
$$

where $K$ is defined by

$$
K=\frac{c^{\beta} a^{\alpha-\beta}}{\Gamma(\beta) \Gamma(\alpha-\beta)}, \alpha>\beta
$$

The log-likelihood function can be written as

$$
\ln L=n \ln K+(\alpha-1) \sum_{i=1}^{n} \ln \left(x_{i}\right)+(\beta-1) \sum_{i=1}^{n} \ln \left(y_{i}\right)-a \sum_{i=1}^{n} x_{i}-c \sum_{i=1}^{n} x_{i} y_{i}
$$

The first-order derivatives of (17) are

$$
\begin{aligned}
\frac{\partial \ln L}{\partial a} & =\frac{n(\alpha-\beta)}{a}-\sum_{i=1}^{n} x_{i} \\
\frac{\partial \ln L}{\partial c} & =\frac{n \beta}{c}-\sum_{i=1}^{n} x_{i} y_{i} \\
\frac{\partial \ln L}{\partial \alpha} & =n \ln a-n \psi(\alpha-\beta)+\sum_{i=1}^{n} \ln \left(x_{i}\right) \\
\frac{\partial \ln L}{\partial \beta} & =n \ln c-n \ln a-n \psi(\beta)+n \psi(\alpha-\beta)+\sum_{i=1}^{n} \ln \left(y_{i}\right)
\end{aligned}
$$


where $\psi(\cdot)$ denotes the psi function given by equation (5).

The maximum likelihood estimators of $(a, c, \alpha, \beta)$ are obtained by equating all the first-order derivatives above to zero. We can show that the MLE's are related by the following equations

$$
\left\{\begin{array}{l}
\frac{n(\hat{\alpha}-\hat{\beta})}{\hat{a}}-\sum_{i=1}^{n} x_{i}=0 \\
\frac{n \hat{\beta}}{\hat{c}}-\sum_{i=1}^{n} x_{i} y_{i}=0 \\
n \ln (\hat{a})-n \psi(\hat{\alpha}-\hat{\beta})+\sum_{i=1}^{n} \ln \left(x_{i}\right)=0 \\
n \ln (\hat{c})-n \ln (\hat{a})-n \psi(\hat{\beta})+n \psi(\hat{\alpha}-\hat{\beta})+\sum_{i=1}^{n} \ln \left(y_{i}\right)=0
\end{array}\right.
$$

\subsection{Fisher information matrix}

The entries above the main diagonal of the FIM of model (2) are obtained by calculating the expectations of the negatives of the following second order derivatives of (17).

$$
\begin{aligned}
\frac{\partial^{2} \ln L}{\partial a^{2}} & =-\frac{n(\alpha-\beta)}{a^{2}} \\
\frac{\partial^{2} \ln L}{\partial a \partial c} & =0 \\
\frac{\partial^{2} \ln L}{\partial a \partial \alpha} & =\frac{n}{a} \\
\frac{\partial^{2} \ln L}{\partial a \partial \beta} & =-\frac{n}{a} \\
\frac{\partial^{2} \ln L}{\partial c^{2}} & =-\frac{n \beta}{c^{2}} \\
\frac{\partial^{2} \ln L}{\partial c \partial \alpha} & =0 \\
\frac{\partial^{2} \ln L}{\partial c \partial \beta} & =\frac{n}{c} \\
\frac{\partial^{2} \ln L}{\partial \alpha^{2}} & =-n \frac{\partial}{\partial \alpha} \psi(\alpha-\beta) \\
\frac{\partial^{2} \ln L}{\partial \alpha \partial \beta} & =n \frac{\partial}{\partial \alpha} \psi(\alpha-\beta) \\
\frac{\partial^{2} \ln L}{\partial \beta^{2}} & =-n\left[\frac{\partial}{\partial \beta} \psi(\beta)+\frac{\partial}{\partial \alpha} \psi(\alpha-\beta)\right]
\end{aligned}
$$

Rev. Bras. Biom., Lavras, v.39, n.2, p.350-361, 2021 - doi: 10.28951/rbb.v39i2.476 
Since all of the second derivatives of (17) are constants, the elements of the FIM are simply the negatives of the second derivatives. Using the identity (8) we can show that the FIM is given by

$$
I=\left(\begin{array}{cccc}
\frac{n(\alpha-\beta)}{a^{2}} & 0 & -\frac{n}{a} & \frac{n}{a} \\
0 & \frac{n \beta}{c^{2}} & 0 & -\frac{n}{c} \\
-\frac{n}{a} & 0 & n \zeta(2, \alpha-\beta) & -n \zeta(2, \alpha-\beta) \\
\frac{n}{a} & -\frac{n}{c} & -n \zeta(2, \alpha-\beta) & n[\zeta(2, \beta)+\zeta(2, \alpha-\beta)]
\end{array}\right)
$$

\section{Application}

In this section we provide an application, with real data, of the results of Section 3. Our data were collected from a series of 730 days of rainfall observations, from July 2009 to July 2011, performed by the Embrapa Trigo Laboratory of Meteorology Applied to Agriculture, (http://www.cnpt.embrapa.br/pesquisa/agromet) located at Passo Fundo, State of Rio Grande do Sul, Brazil (latitude: 28 $15^{\prime} 46^{\prime \prime}$ S; longitude: $52^{\circ} 24^{\prime} 24^{\prime \prime} \mathrm{W}$, altitude: $684 \mathrm{~m}$ ). Our random variables are pairs $\left(X_{j}, Y_{j}\right), j=1, \ldots, n$, where $X_{j}$ is the number of consecutive rainfall days and $Y_{j}$ the number of consecutive non-rainfall days. More specifically, suppose that the first of the 730 days were a rainfall day, then we count the number of consecutive rainfall days and call $X_{1}$; the number of consecutive following non-rainfall days is $Y_{1}$, and so on. Following this procedure, we got $n=118$ climatic periods (observations).

Given a sample $\left(x_{1}, y_{1}\right),\left(x_{2}, y_{2}\right) \ldots\left(x_{n}, y_{n}\right)$ of $(X, Y)$, the maximum likelihood estimators of the parameters of the bivariate model gamma beta of the second kind and standard errors are:

$\hat{a}=0.8654(0.1183) ; \hat{c}=0.1899(0.0269) ; \hat{\alpha}=3.3920(0.2965) ; \hat{\beta}=1.2842(0.1503)$.

The standard errors of the parameter was computed by inverting the expected information matrix. The corresponding estimates of the elements of the FIM of the model are given by

$$
I=\left(\begin{array}{cccc}
332.29 & 0 & -136.42 & 136.42 \\
0 & 4241.53 & 0 & -624.34 \\
-136.42 & 0 & 71.31 & -71.31 \\
136.42 & -624.34 & -71.31 & 207.45
\end{array}\right)
$$

The variance-covariance matrices of parameters of distribution is obtained by calculating the inverse of the (18), resulting in 


$$
\operatorname{Cov}(\hat{a}, \hat{c}, \hat{\alpha}, \hat{\beta})=\left(\begin{array}{cccc}
0.0140 & -- & -- & -- \\
0.0000 & 0.0007 & -- & -- \\
0.0268 & 0.0033 & 0.0879 & -- \\
0.0000 & 0.0033 & 0.0226 & 0.0226
\end{array}\right)
$$

The calculations of the FIM and its inverse matrix were obtained by using the software R (R CORE TEAM, 2019) and its package VGAM (YEE et al., 2015) .

\section{Conclusion}

The estimators of the parameters of the models were discussed by using the maximum likelihood method. Explicit expressions for the FIM's for Crovelli's and gamma beta II bivariate distributions were derived. The expressions involve the Hurwitz zeta function. The use of the results to calculate the standard error of the maximum likelihood estimators were also illustrated.

\section{Acknowledgments}

The authors would like to thank the reviewers and editors for their valuable suggestions and contributions to this paper. It was of great help.

SILVA, A. P. C. M.; DINIZ, A. C. Matriz de Informação de Fisher para as distribuições gama bivariada de Crovelli e gama beta tipo II. Rev. Bras. Biom., Lavras, v.39, n.1, p.350-361, 2021.

- RESUMO: Neste artigo, um sistema de equações não lineares para os estimadores de máxima verossimilhança, bem como as formas exatas da matriz de informação de Fisher para as distribuições gama bivariada de Crovelli e gama beta do tipo II, são fornecidos. Uma aplicação dos resultados aos dados pluviométricos da cidade de Passo Fundo é fornecida.

- PALAVRAS-CHAVE: Distribuição bivariada; estimador de máxima verossimilhança; função zeta de Hurwitz; matriz Hessiana.

\section{References}

ALI, M.; NADARAJAH, S. Information matrices for normal and Laplace mixtures. Information Sciences, v.177, p.947-955, 2007.

FISCHER, A. Fisher information and Cramér-Rao bound for unknown systematic errors. Journal of the International Measurement Confederation, v.113, p.131-136, 2018. 
BALAKRISHNAN, N.; LAI, C. D. Continuos bivariate distributions. New York: Springer, 2009, 684p.

BARROS, P. S. N.; BRITO, C. C. R.; REGO, L. C.; OLIVEIRA, W. R.; GOMES, S. F. A new class of generalized Weibull distributions. Revista Brasileira de Biometria, v.35, n.2, p.319-347, 2017.

BEALS, R.; WONG, R. Special functions . New York: Cambridge, 2010, 456p.

COX, D.R.; HINKLEY, D.V. Theoretical Statistics. London: Chapman and Hall, 1974, 528p.

GUPTA, A. K.; NADARAJAH, S. Information matrices for some bivariate pareto distributions. Applied mathematics and computation, v.184, n.2, p.1069-1079, 2007.

LIU, W.; WANG, Y.; XIE, W. Fisher information matrix, rao test, and wald test for complex-valued signals and their applications. Signal Processing, v.94, p.1-5, 2014.

LOUZADA, F.; ELBATAL, I.; GRANZOTTO, D. C. T. The beta exponentiated weibull geometric distribution: modeling, structural properties, estimation and an application to a cervical intraepithelial neoplasia dataset. Revista Brasileira de Biometria, v.36, n.4, p.942-967, 2018.

LV, S. General fisher information matrices of a random vector. Advances in Applied Mathematics, v.89, p.18-40, 2017.

LY, A.; MARSMAN, M.; VERHAGEN, J.; GRASMAN, R. P A tutorial on fisher information. Journal of Mathematical Psychology, v.80, p.40-55, 2017.

NADARAJAH, S. Information matrix for the bivariate gumbel distribution. Applied mathematics and computation, v.172, n.1, p.394-405, 2006a.

NADARAJAH, S. Fisher information for the elliptically symmetric Pearson distributions. Applied Mathematics and Computation,v. 178, p.195-206, 2006b.

OLDHAM, K. B.; MYLAND, J.; SPANIER, J. An atlas of functions: with equator, the atlas function calculator. New York: Springer Vergland, 2009, 784p.

PARK, J. S.; KIM, T. Y. Fisher information matrix for a four-parameter kappa distribution. Statistics \& probability letters, v.77, n.13, p.1459-1466, 2007.

QIAN, L. The fisher information matrix for a three-parameter exponentiated weibull distribution under type ii censoring. Statistical Methodology, v.9, n.3, p.320-329, 2012.

R CORE TEAM (2019). R: A Language and Environment for Statistical Computing . R Foundation for Statistical Computing, Available from: https://www.Rproject.org/

SILVA, A. P. C. M.; RODRIGUES, J. A.; CHAVES, L. M.; SOUZA, D. J. Sums, products and ratios for Crovelli's bivariate gamma distribution. Water resources management, v.27, n.5, p.1363-1376, 2013a. 
SILVA, A. P. C. M.; RODRIGUES, J. A.; CHAVES, L. M.; SOUZA, D. J. Distribuição bivariada gama beta II: soma, produto e proporção das variáveis componentes. TEMA- Trends in Applied and Computational Mathematics, v.14, n.1, p.95-108, 2013b.

YEE, T. W.; STOKLOSA, J.; HUGGINS, R. M. The VGAM package for capturerecapture data using the conditional likelihood. Journal of Statistical Software, v.65, n.5, p.1-33, 2015.

Received on 20.05.2020.

Approved after revised on 17.08.2020. 